\title{
Flow field and noise characteristics of manifold in natural gas transportation station
}

\author{
Zhongya $\mathrm{Su}^{1}$, Enbin $\mathrm{Liu}^{1}{ }^{1}$, Yawen $\mathrm{Xu}^{2}$, Ping $\mathrm{Xie}^{3}$, Chen Shang ${ }^{3}$, and Qiyong Zhu ${ }^{3}$ \\ ${ }^{1}$ Petroleum Engineering School, Southwest Petroleum University, Chengdu 610500, China \\ ${ }^{2}$ PetroChina Southwest Oil \& Gasfield Company, Chengdu 610000, China \\ ${ }^{3}$ PetroChina West Pipeline Company, Urumchi 830001, China
}

Received: 3 February 2019 / Accepted: 12 June 2019

\begin{abstract}
Manifolds play a role of pressure balance, buffering and rectification for different branch pipelines, the flow noise of manifolds has been a serious problem all this time in natural gas transmission station. By changing the number of outlet pipes of manifolds and the different positions of intake pipes, the distribution of the Sound Pressure Level (SPL) of the manifold flow noise is analyzed based on the Ffowcs WilliamsHawkings (FW-H) acoustic analogy theory and Large Eddy Simulations (LESs). The three-dimensional simulation analysis of the flow field shows that pressure pulsation is the mainly source of manifold noise, as the number of outlet pipe increases, the SPLs of fluid dynamic noise at the end of inlet pipes are significantly reduced by about $10 \mathrm{~dB}$ on average, when the inlet and outlet piping are oppositely connected, the SPL is $2 \mathrm{~dB} \sim 3 \mathrm{~dB}$ lower than that in staggered connections. An expansion-chamber muffler is designed with the analysis of its noise reduction effect, the results show that after the muffler is installed, the noise reduction in the lowfrequency ranges reaches up to $37.5 \mathrm{~dB}$, which controls the maximum noise to around $82 \mathrm{~dB}$.
\end{abstract}

\section{Introduction}

For the past few years, with the rapid growth of natural gas demand in China, the annual throughput of gas pipelines has been constantly increasing. Consequently, many stations experienced an obvious problem of noise pollution. The continuous strong station noise not only can cause damaging effects on people's health, but also covers the danger singer, resulting in the distortion of the measuring instruments of pipelines, bringing severe harm to the production safety [1, 2].

Though, there are a variety of noise sources in natural gas station, which causes various types of noise, it is found through field investigation that the mainly noise is the fluid dynamic noise generated by the process pipeline and equipment. Natural gas transmission includes multiple links, when the station is pressure-regulated and diverted, the flow state of the medium in the pipe is prone to abrupt change, forming disturbance, eddy current and vortex resistance, the friction along the pipe walls increases, and the energy spreads out in sound waves. So, the aerodynamic noise is thus generated. Especially for large-scale gas stations, due to the dizzying number of intake and distribution pipelines, the noise problem is of great serious and can not be evaded [3].

\footnotetext{
* Corresponding author: enbin.liu@swpu.edu.cn
}

In the 1950s, the research of flow noise made great progress with the landmark event of the establishment of Lighthill's acoustic analogy theory $[4,5]$. Lighthill equation describes the sound field and the flow field separately, and succeeds in resolving the problem of the sound caused by moving fluid. Thereafter, experts and scholars all over the word restudied the Lighthill equation and developed their respective theories. Curle considered the influence of static solid boundaries [6]. Powell proposed the theory of vortex sound [7]. Williams and Hawkings extended Curle's theory to consider the influence of nonstationary solid boundaries and obtained the famous Ffowcs Williams-Hawkings (FW-H) equation [8]. Lauchle further developed Curle's theory and proposed the radiated noise model of boundary layer transition [9]. Goldstein analyzed the noise mechanism of moving transmission medium and obtained the universally applicable Lighthill equation [10]. With the development of numerous theories, the understand of noise mechanism has been more profound.

Early researches on aerodynamic noise were dominated by the theoretical analysis and experiments. Ma et al. conducted in-depth theoretical and experimental research on the noise production of subsonic and supersonic jets and obtained the relationship between turbulent jet noise and pressure [11-13]. Wu studied the wave-vortex interaction and the mechanism of vortex emission and absorption of sound waves $[14,15]$. With the development of computer 
technology, numerical simulation is realized, and various turbulence calculation models are widely used. Mankbadi et al. applied the LES to the study of jet noise and predicted the far-field sound field $[16,17]$. Wu and Zhang investigated the 3-D separate flow of butterfly valve, gate valve and ball valve in the water piping and proved that the flow noise at valve downstream is bigger than that at the valve upstream, and vortex motion is the mainly source of valve noise [18]. Tan and Wang analyzed the velocity field in downstream gas pipe of manifold and obtained the reasonable velocity field distribution [19]. Liu et al. did several aggressive studies on aerodynamic noise, which included the analysis of the factors influence the noise of upright venting pipes, elbows and manifolds [20-23].

The manifold is widely used in natural gas station and plays a role of pressure balancing, buffering and rectification for different branch pipelines. The pipe diameter of manifold is much larger than that of most pipe segments, and it usually works under high pressure. When natural gas flows through a manifold, high level aerodynamic noise is easily produced due to the influence of eddy current disturbance [24-26]. In this paper, the flow field inside the manifold is simulated. Meanwhile, an expansion-chamber muffler is designed. The results of numerical simulations show that the noise inside the manifold is decreased obviously after the muffler is installed.

\section{Numerical methods}

\subsection{Mathematical model}

The FW-H function is widely used in aerodynamic acoustic. It is derived from the Lighthill's acoustic analogy theory using the generalized function method and satisfies the problem of flow noise caused by the interaction between a nonstationary solid boundary and a fluid medium. Suppose the moving object surface in the fluid can be described by $c\left(x_{i}, t\right)=0$, and the area inside the object surface is represented by $c\left(x_{i}, t\right)<0$, the area outside the object surface is represented by $c\left(x_{i}, t\right)>0$. The $\mathrm{FW}-\mathrm{H}$ function is shown below:

$$
\begin{aligned}
\frac{1}{a_{0}^{2}} \frac{\partial^{2} p^{\prime}}{\partial t^{2}}-\nabla^{2} p^{\prime}= & \frac{\partial^{2}}{\partial x_{i} \partial x_{j}}\left\{T_{i j} H(c)\right\} \\
& -\frac{\partial}{\partial x_{i}}\left\{\left[P_{i j} n_{j}+\rho u_{i}\left(u_{n}-v_{n}\right)\right] \delta(c)\right\} \\
& +\frac{\partial}{\partial t}\left\{\left[\rho_{0} v_{n}+\rho\left(u_{n}-v_{n}\right)\right] \delta(c)\right\},
\end{aligned}
$$

where $p^{\prime}$ is the sound pressure at far field $(\mathrm{Pa}) ; a_{0}$ is the local sound speed $(\mathrm{m} / \mathrm{s}) ; \rho_{0}$ is the fluid density $\left(\mathrm{kg} / \mathrm{m}^{3}\right)$; $u_{n}$ is the flowing medium velocity component $(\mathrm{m} / \mathrm{s}) ; v_{n}$ is the surface velocity component $(\mathrm{m} / \mathrm{s}) ; T_{i j}$ is the Lighthill stress tensor $(\mathrm{Pa}) ; \delta(c)$ is the Dirac function, and $H(c)$ is the Heaviside function. It satisfies:

$$
H(c)=\left\{\begin{array}{l}
1, c\left(x_{\mathrm{i}}, t\right)>0 \\
0, c\left(x_{\mathrm{i}}, t\right)<0
\end{array}, \quad \delta(c)=\frac{\partial H(c)}{\partial c}\right.
$$

In Large Eddy Simulation (LES) theory, the motion is divided into vortices of different sizes and the small-scale vortices are thought to be less affected by the flow fields. By filtering the $\mathrm{N}-\mathrm{S}$ equations in physical space or wave number, the equation can be obtained as follows:

$$
\frac{\partial}{\partial t}\left(\rho \bar{u}_{i}\right)+\frac{\partial}{\partial x_{j}}\left(\rho \bar{u}_{i} \bar{u}_{j}\right)=\frac{\partial}{\partial x_{j}}\left(u \frac{\partial \bar{u}_{i}}{\partial x_{j}}\right)-\frac{\partial \bar{p}}{\partial x_{j}}-\frac{\partial \tau_{i j}}{\partial x_{j}},
$$

where $\tau_{i j}$ is the subgrid stress (MPa); $u_{i}$ and $u_{j}$ are velocity components of the fluid $(\mathrm{m} / \mathrm{s}) ; x_{j}$ is the length component $(\mathrm{m}) ; t$ is the time(s).

In order to more intuitively analyze the Sound Pressure Level (SPL) and its distribution inside the manifold, the pressure pulsation signal receivers were placed, and the time domain pressure pulsations measured by each receiver were converted into the frequency domain using the Fast Fourier Transform (FFT).

$$
p(f)=\frac{1}{2 \pi} \int_{-\infty}^{+\infty} p(t) e^{\mathrm{itf}} \mathrm{d} t,
$$

where $p(t)$ is the pressure function in time domain; $p(f)$ is the pressure function in frequency domain; $e$ is the base of the natural logarithm; $i$ is the imaginary unit that has the property $i^{2}=-1 ; f$ is the frequency $(\mathrm{Hz}) ; t$ is the time (s).

The SPL can be written as:

$$
\mathrm{SPL}=20 \log \frac{p_{\text {rms }}}{p_{\text {ref }}},
$$

where the SPL is measured $(\mathrm{dB}), p_{\text {rms }}$ is the pressure pulsation $(\mathrm{Pa}) ; p_{\text {ref }}$ is the reference $\mathrm{SPL}$, and the value is $2 \times 10^{-5} \mathrm{~Pa}$.

\subsection{Numerical calculation}

With the modulation of the fluid field near the manifold by ANSYS Fluent, the establishment of the model is done by transferring the distribution of fluid to a sound source at the surface of the manifold by the FW-H theory. The steady-state condition of the $k-\varepsilon$ equation is selected as the initial condition of the LES transient simulation. To speed up the convergence, PISO is chosen as the iterative method, and the time step is $2.5 \times 10^{-4} \mathrm{~s}$.

\section{Result and discussions}

Using Pro/Engineer to establish 3D models of the manifold. The manifold diameter $D_{m}=600 \mathrm{~mm}$, manifold length $L_{m}=4000 \mathrm{~mm}$, inlet pipe diameter $D_{\text {in }}=200 \mathrm{~mm}$, inlet pipe length $L_{\text {in }}=800 \mathrm{~mm}$, outlet pipe diameter $D_{\text {out }}=$ $200 \mathrm{~mm}$, outlet pipe length $L_{\text {out }}=800 \mathrm{~mm}$. The fluid inside the manifold is methane, and the sound velocity $c_{0}=447 \mathrm{~m} / \mathrm{s}$, temperature $T=293 \mathrm{~K}$, dynamic viscosity $\mu=1.1067 \times 10^{-5} \mathrm{Pas}$. The boundary conditions are velocity-inlet and pressure-outlet. The fluid velocity satisfies the no-slip boundary condition, which means that the velocity at the pipe wall is 0 . 

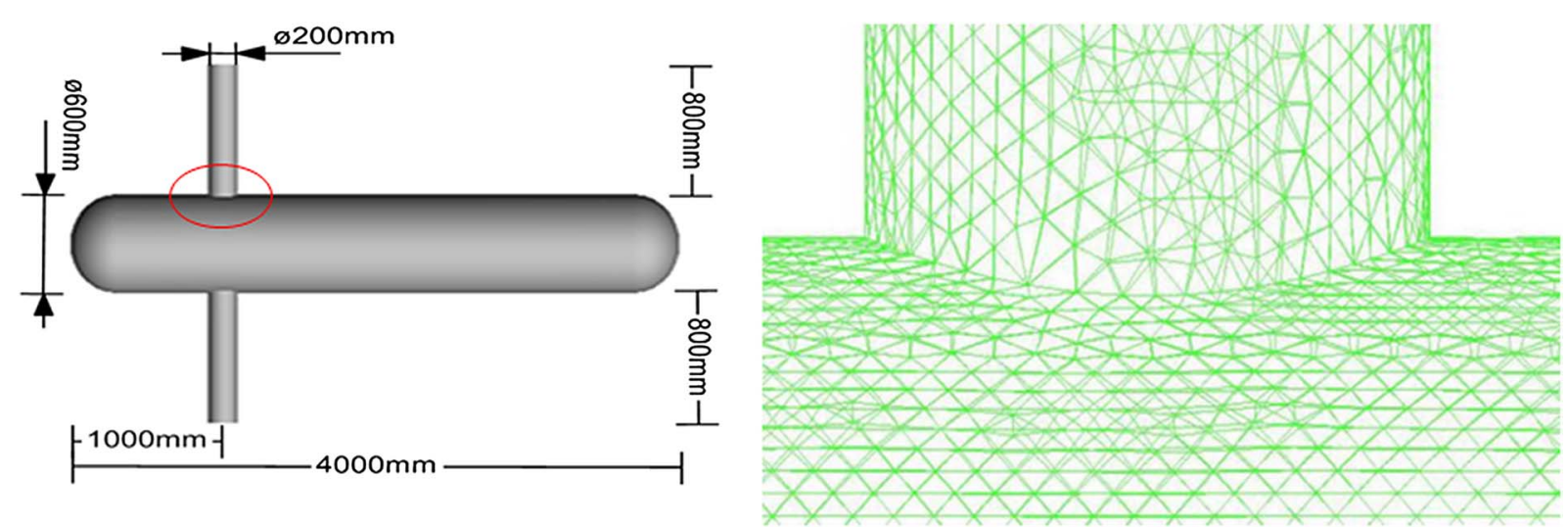

Fig. 1. The geometric model and grid division of the manifold with one inlet and one outlet.

Taking the typical oppositely connected manifold with one inlet and one outlet as an example, the manifold model and grid division are as shown in Figure 1.

In the usage of LES, the calculation error caused by the grid number and the time step need to be eliminated. In this study, the effectiveness of this method is verified by comparing the experimental results under four different grid number (about 1500 000, 3400 000, 4600000 and $5800000)$.

As we can see in Figure 2, with the grid number increase, the SPL changes little. And considering the amount of calculation, in this study, 4600000 of the computational grid number has been chosen.

Similarly, for time independency, the time step has been selected as $0.00025 \mathrm{~s}$.

\subsection{Field and acoustic field}

Studies have shown that there are two main causes of pipeline noise, one is the pressure pulsation caused by fluid flow, and the other is the friction, collision and disturbance generated by the internal medium motion inside the pipe $[27,28]$. The pressure pulsation inside the manifold is generally generated by the eddy current motion. In Fluent post-processing, the eddy current motion can be analyzed indirectly through the velocity field and the acoustic power level distribution. The position where the eddy motion is likely to occur, the pressure pulsation is large, and the noise is easily produced.

Taking the oppositely connected manifold with one inlet and one outlet as an example, the velocity field and acoustic power level distribution are shown in Figures 3 and 4.

Figure 3 shows that, when flowing through the manifold with one inlet and one outlet, the velocity at both ends of the manifold is small, while the velocity at the inlet and outlet pipes is relatively large, among which the velocity at the outlet trachea is $10 \% \sim 20 \%$ higher than that at the inlet pipe. Because of the guiding of the outlet pipe, the fluid inside the manifold ejected by the intake pipe moves toward the outlet as it approaches the pipe wall. And combined with the influence of the boundary layer near the manifold wall, eddies appear and became the source of the fluid dynamic noise. Moreover, the manifold diameter is much

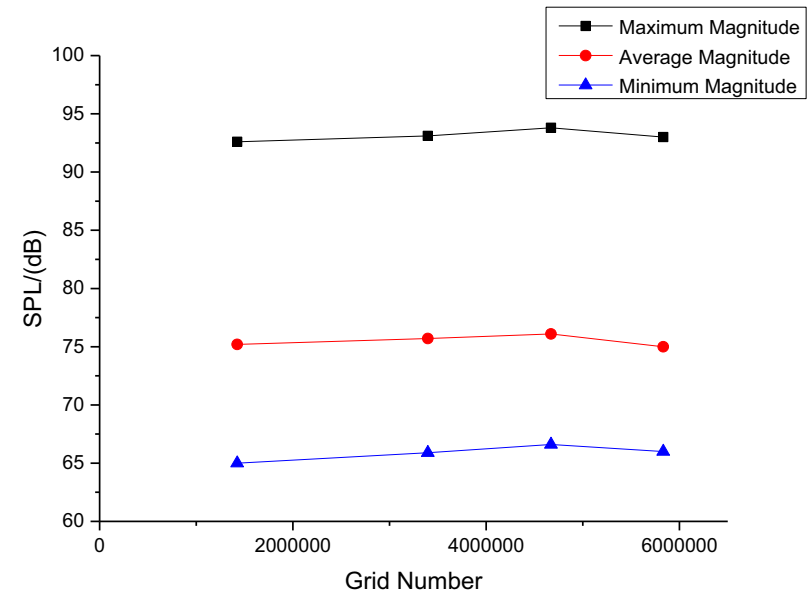

(a) SPL distribution at the end of inlet pipe

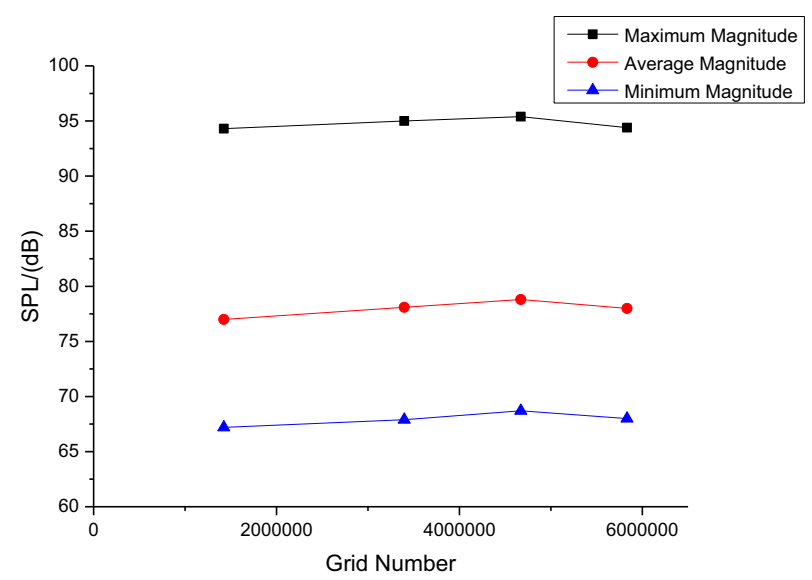

(b) SPL distribution at the head of outlet pipe

Fig. 2. SPL distribution with different grid number. (a) SPL distribution at the end of inlet pipe. (b) SPL distribution at the head of outlet pipe.

larger than that of the inlet and outlet pipes. When gas flows in the manifold, there is a sudden change in velocity at the head of the outlet pipe. Causing greater pressure pulsation, and the noise is generated in the manifold. 


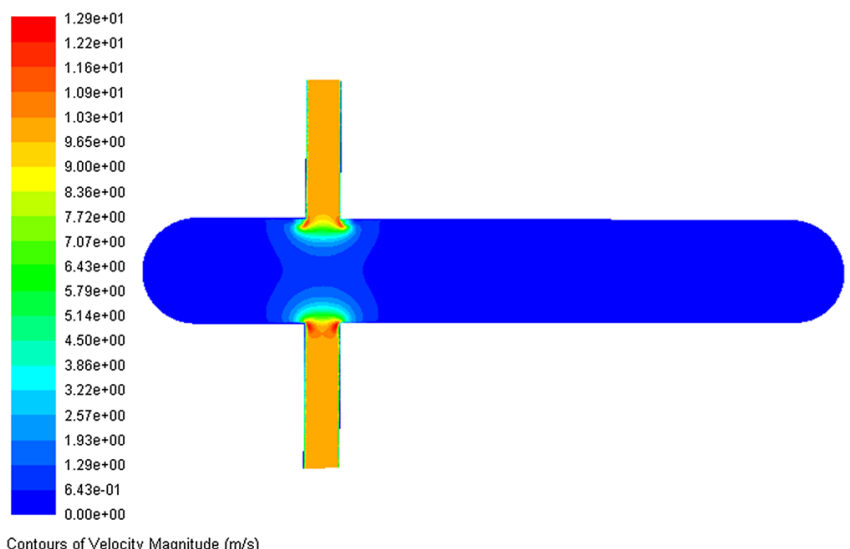

Fig. 3. Velocity contours in the manifold.

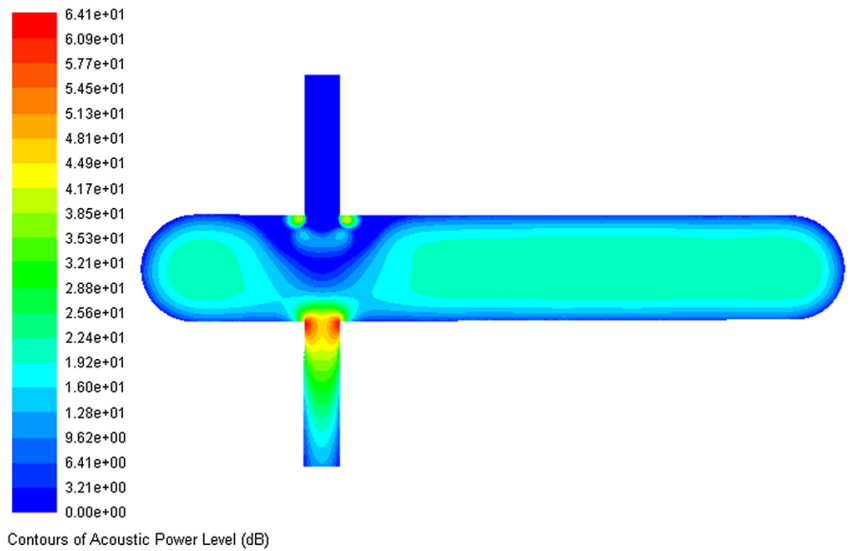

Fig. 4. Acoustic power level distribution in the manifold.

The location of the maximum noise can be clearly seen from Figure 4, that is, the first section of the manifold's outlet pipe. For the oppositely connected manifold with one inlet and one outlet, the flow of the medium near outlet pipe is unstable and cannot be smoothly transitioned. Therefore forming strong eddies and generating noise. Meanwhile, the flow is relatively stable and the velocity is low at both ends of the manifold, which makes the flow noise must also be low.

\subsection{Sound pressure level}

In Figure 5, pressure pulsation signal monitoring points were placed inside the manifold, from which we can analyze the SPL and its distribution more intuitively. By setting up these monitoring points, the time-domain curve of pressure pulsation at each monitoring point can be conveniently obtained. However, it is not enough to observe the structural characteristics of the manifold spectrum only through the time-domain curve of the pulsating pressure. At this time, the FFT in Fluent acoustic module is utilized to convert the time-domain curve of pulsating pressure into the frequency-domain pulsating pressure.

The pressure pulsations in the frequency domain are shown in Figure 6. It shows that, the SPL inside the

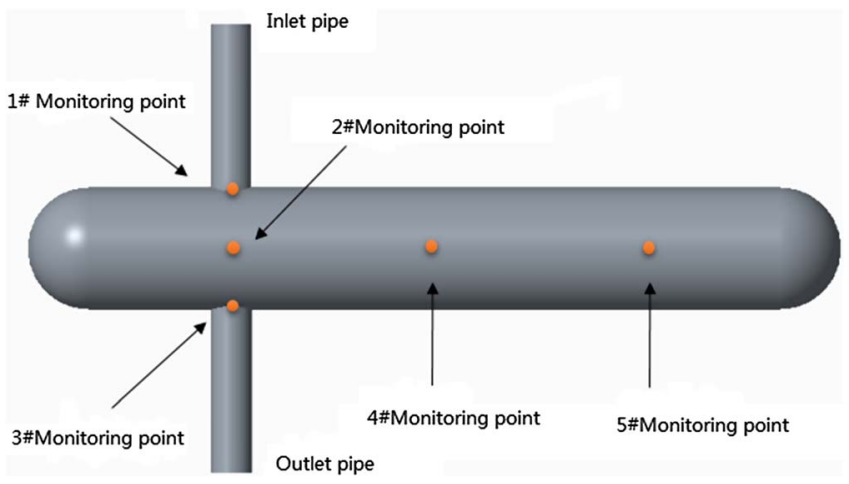

Fig. 5. The sketch of manifold's pressure-monitoring points.

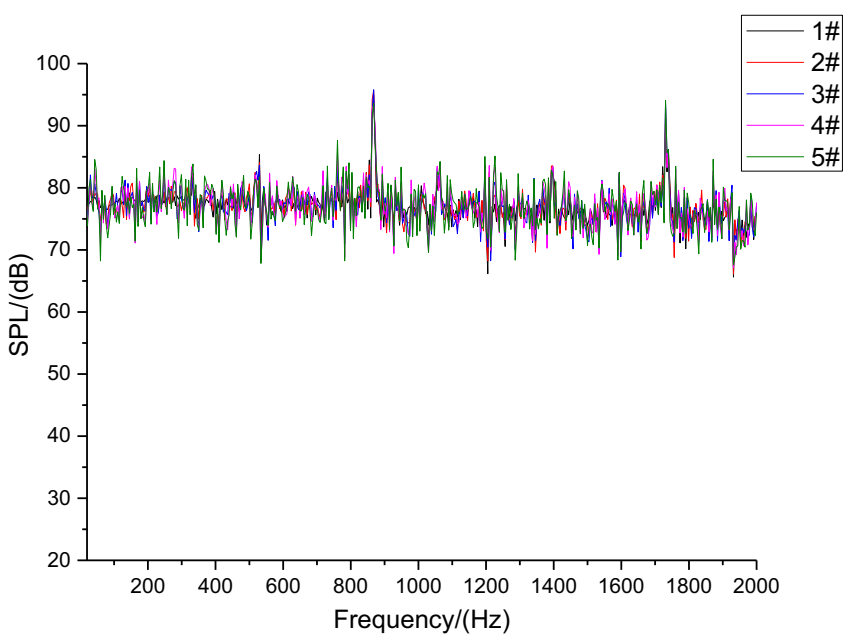

Fig. 6. The SPL distribution of the manifold with one inlet and one outlet.

manifold is relatively stable with a small range of fluctuation. The SPL is mostly distributed among $70 \mathrm{~dB} \sim 80 \mathrm{~dB}$, where the maximum value is $96.4 \mathrm{~dB}$, the minimum value is $65.1 \mathrm{~dB}$, and the average SPL is $75.8 \mathrm{~dB}$. When the frequency is around $850 \mathrm{~Hz}, 1200 \mathrm{~Hz}$ and $1800 \mathrm{~Hz}$, the SPL inside the manifold is obviously fluctuated, among which the SPL at frequency near $850 \mathrm{~Hz}$ and $1800 \mathrm{~Hz}$ is higher than the industrial regulation. Therefore, attention should be paid to the excessive noise near these frequency bands.

By changing the number of outlet pipes of manifolds and the different positions of intake pipes, the flow field and SPL of these manifolds are compared. Through the FFT, the pressure pulsations in the frequency domain at the end of the inlet pipes are obtained. Among them, 1 and 2 are manifolds with one inlet and one outlet, 3 and 4 are manifolds with one inlet and two outlets, 5, 6 and 7 are manifolds with one inlet and three outlets. The branches above the manifolds are the intake pipes, and the lower branches are the outlet pipes. Each inlet and outlet pipe is distributed at $1 / 4,1 / 2,3 / 4$ of the pipe segment, the inlet pipe of manifold 6 is located at $3 / 8$ of the pipe section, as shown in Table 1.

The simulation results of manifold 2 have been shown in Figures 3 and 4. In Figures 7 and 8, the simulation results of 
Table 1. The different structures of manifolds.

\begin{tabular}{|c|c|c|c|c|c|c|c|}
\hline \multirow[t]{2}{*}{$\begin{array}{l}\text { Manifold } \\
\text { structures }\end{array}$} & \multicolumn{2}{|c|}{$\begin{array}{c}\text { One inlet and one } \\
\text { outlet }\end{array}$} & \multicolumn{2}{|c|}{$\begin{array}{c}\text { One inlet and two } \\
\text { outlets }\end{array}$} & \multicolumn{3}{|c|}{ One inlet and three outlets } \\
\hline & $\stackrel{\|}{\|}$ & $\frac{\pi}{\pi}$ & p & $\Perp_{\|\|}$ & $\oiiint_{\|1\|}$ & $\underbrace{}_{\|\|}$ & $\frac{1}{1 \|}$ \\
\hline Numbers & 1 & 2 & 3 & 4 & 5 & 6 & 7 \\
\hline
\end{tabular}
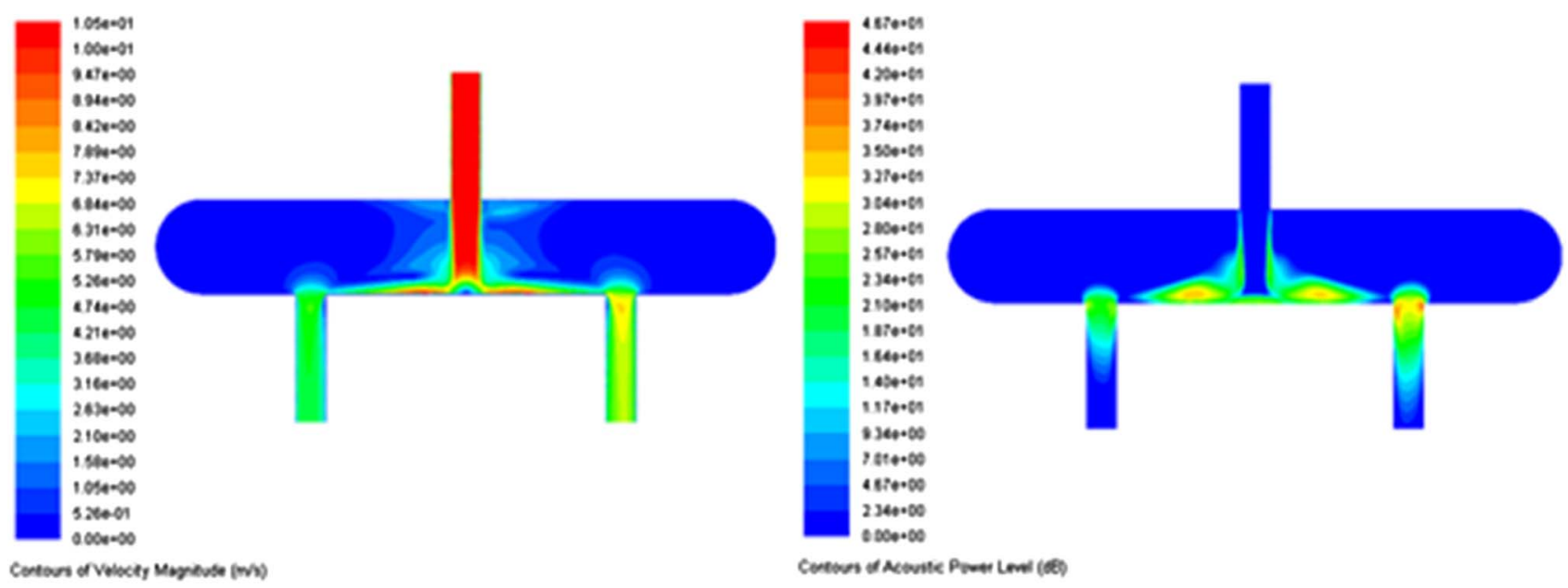

Fig. 7. Velocity contours and acoustic power level distribution of manifold 3.

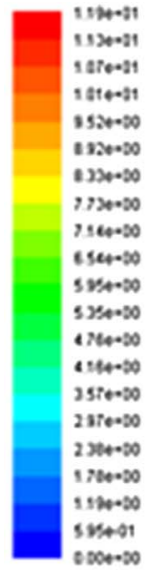

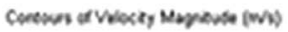

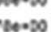

$\sec 20$

N401

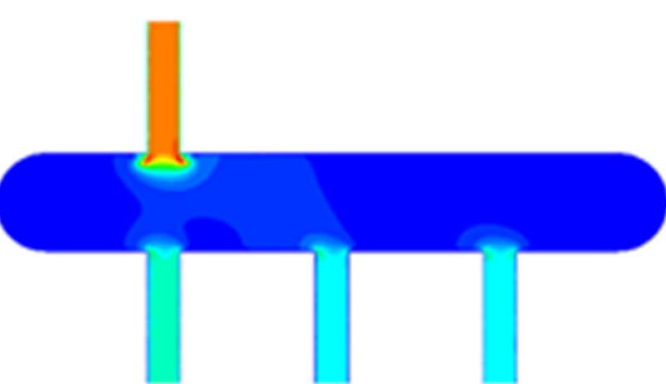

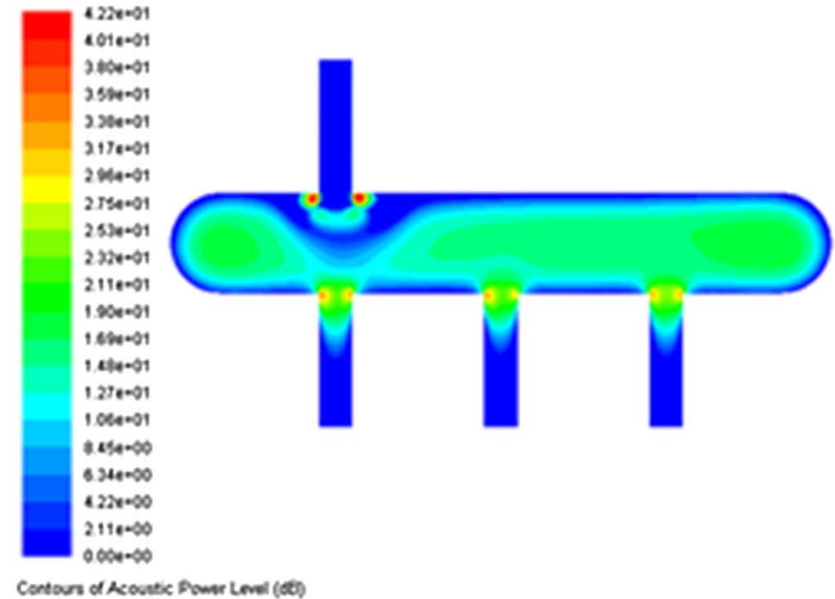

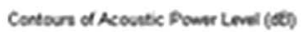

Fig. 8. Velocity contours and acoustic power level distribution of manifold 5.

manifold 3 and manifold 5 are presented. Through the comparative analysis of these simulation results, we can easily find that although the manifold structure is different, the causes of flow noise in manifolds are similar. Therefore, the general rule of noise generation in the manifold can be obtained, that is, if the flow state of the medium is abrupt, and the flow cannot be smoothly transitioned, strong eddies will be generated at the position where the flow velocity is abrupt, and the faster the flow rate, the more irregular the air passage, the easier it is to form eddies. Eddy currents result in pressure pulsations. So, it can be reasonably inferred that the position where the gas flow state is abrupt, the noise inside the manifold is serious.

Figure 9 shows the pressure pulsations in the frequency domain, from which we can see that, with the increase of the number of outlet pipes, the SPL drops significantly, and the positions of the intake pipes are different, the SPL is also different. For the manifold with one inlet and one outlet, the SPL is mostly distributed among $70 \mathrm{~dB} \sim 80 \mathrm{~dB}$, but the SPL of the manifold 2 is $2 \mathrm{~dB} \sim 3 \mathrm{~dB}$ lower than that of the manifold 1 . For the manifold with one inlet and two outlets, the SPL is mostly distributed among 


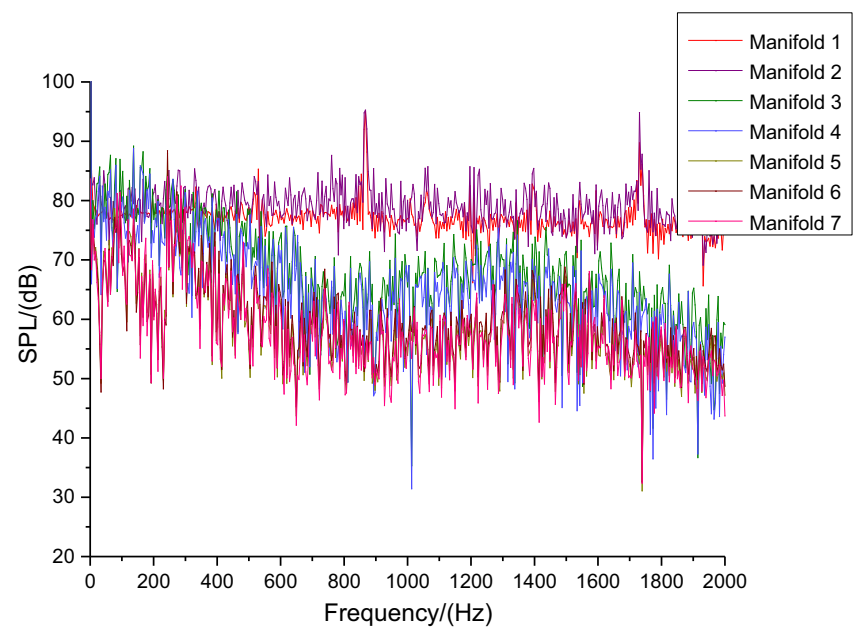

Fig. 9. The SPLs at the end of the inlet pipes.

$60 \mathrm{~dB} \sim 80 \mathrm{~dB}$, and the SPL of the manifold 4 is $2 \mathrm{~dB} \sim 3 \mathrm{~dB}$ lower than that of the manifold 3 . In the frequency range of $20 \mathrm{~Hz} \sim 800 \mathrm{~Hz}$, the SPL drops significantly. For the manifold with one inlet and three outlets, the SPL is mostly distributed among $50 \mathrm{~dB} \sim 70 \mathrm{~dB}$, and the SPL of the manifold 6 is $1 \mathrm{~dB} \sim 2 \mathrm{~dB}$ lower than that of the manifold 5 , the SPL of the manifold 7 is $1 \mathrm{~dB} \sim 2 \mathrm{~dB}$ lower than that of the manifold 6 . In the frequency bands of $100 \mathrm{~Hz} \sim 300 \mathrm{~Hz}$, the SPL reaches the maximum. Therefore, an effective method to reduce the SPL inside the manifold is to increase the number of outlets, however, if the on-site process is not permitted, it is wise to select a reasonable manifold structure, which can also effectively reduce noise.

\section{Expansion-chamber muffler design and evaluation}

Due to the inherent characteristics in reducing low and middle frequency, small pressure loss and simple geometry, the expansion-chamber muffler is being extensively used in natural gas transmission station.

Figure 10 shows the muffling principle of expansionchamber muffler, that is, the expansion cavity causes reflection of sound waves, which hinders the normal propagation of sound energy. According to the continuous conditions of sound pressure, volume and velocity at the variable cross section and the law of conservation of energy, the sound pressure transmission coefficient can be obtained:

$$
t_{p}=\frac{2 S_{1}}{S_{2}+S_{1}}
$$

where $t_{p}$ is the sound pressure transmission coefficient; $S_{1}$ is the area of the pipe section; $S_{2}$ is the area of the expansion section.

The sound intensity transmission coefficient can be written as:

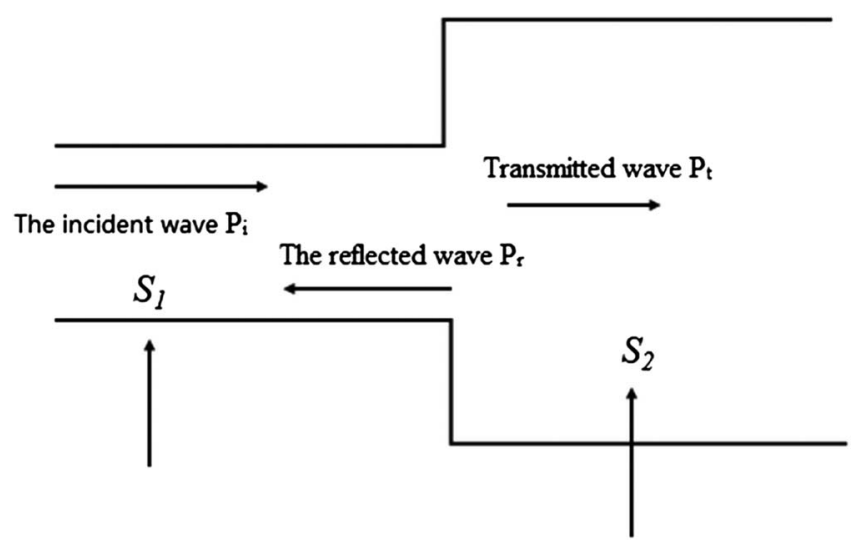

Fig. 10. Muffler schematic.

$$
t_{I}=\frac{S_{2}}{S_{1}}\left(t_{p}\right)^{2}
$$

where $t_{I}$ is the sound intensity transmission coefficient.

The silencing effect of the muffler can be written as:

$$
\begin{aligned}
\mathrm{TL} & =10 \log \frac{1}{t_{I}}=10 \log \frac{\left(S_{1}+S_{2}\right)^{2}}{4 S_{1} S_{2}} \\
& =10 \log \frac{\left(1+S_{2} / S_{1}\right)^{2}}{4 S_{2} / S_{1}}=10 \log \frac{(1+m)^{2}}{4 m},
\end{aligned}
$$

where TL is the noise reduction of the muffler; $m$ is the expansion ratio of expansion cavity.

The silencing effect of the expansion-chamber muffler mainly depends on the ratio of its cross-sectional area $m=S_{2} / S_{1}$, that is, the expansion ratio of expansion cavity, while the length of expansion cavity has little influence on the silencing performance of the muffler. Generally speaking, increasing the expansion ratio is an important way to improve the muffler's silencing performance, however, the expansion ratio is not as big as possible, in practice, it is usually $5<m<20[29,30]$.

In the case that the length of expansion cavity remains unchanged, three types of expansion-chamber mufflers with expansion ratio of $m=6, m=9$ and $m=16$ are selected respectively to compare their silencing effect (Fig. 11). The main design parameters are as follows: muffler diameter $D=200 \mathrm{~mm}$, muffler length $L=1000 \mathrm{~mm}$, expansion cavity diameter $D_{\mathrm{ex}}=D \sqrt{m}$, expansion cavity length $L_{\text {ex }}=600 \mathrm{~mm}$.

The numerical simulation of muffler silencing effect is performed with the typical oppositely connected manifold with one inlet and one outlet, and the muffler is connected to the inlet pipe through a flange, as shown in Figure 12. The SPL of manifolds with no muffler and three different expansion ratios mufflers are shown in Figure 13.

As shown in Figure 13, when there is no muffler, the SPL inside the manifold is mostly distributed among $75 \mathrm{~dB} \sim 85 \mathrm{~dB}$, and even more than $90 \mathrm{~dB}$ in some frequency bands. After the muffler is installed, the SPL inside the manifold shows a significant downward trend, especially in the low frequencies $(20 \mathrm{~Hz} \sim 500 \mathrm{~Hz})$. When the muffler 

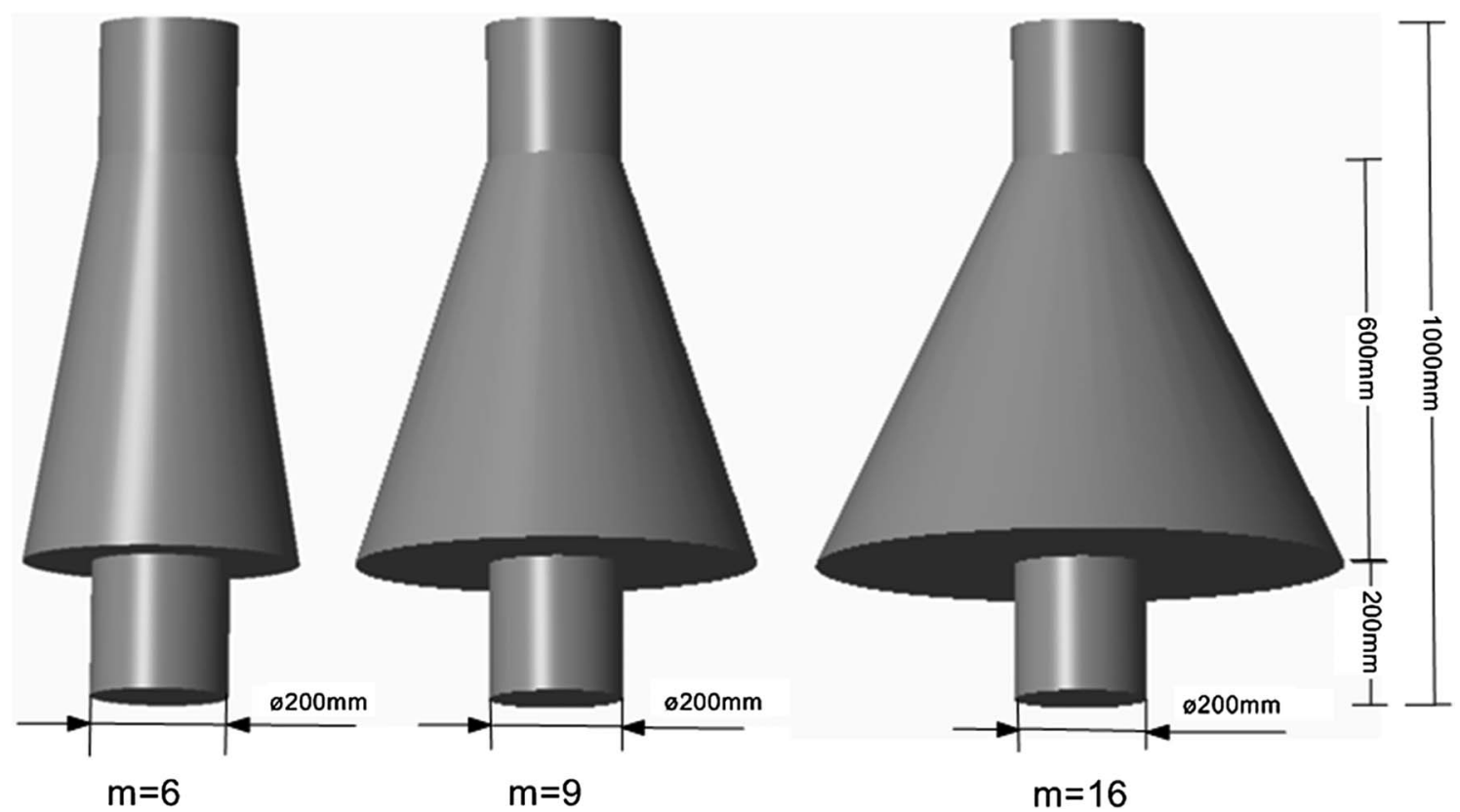

Fig. 11. The expansion-chamber mufflers with different expansion ratios.

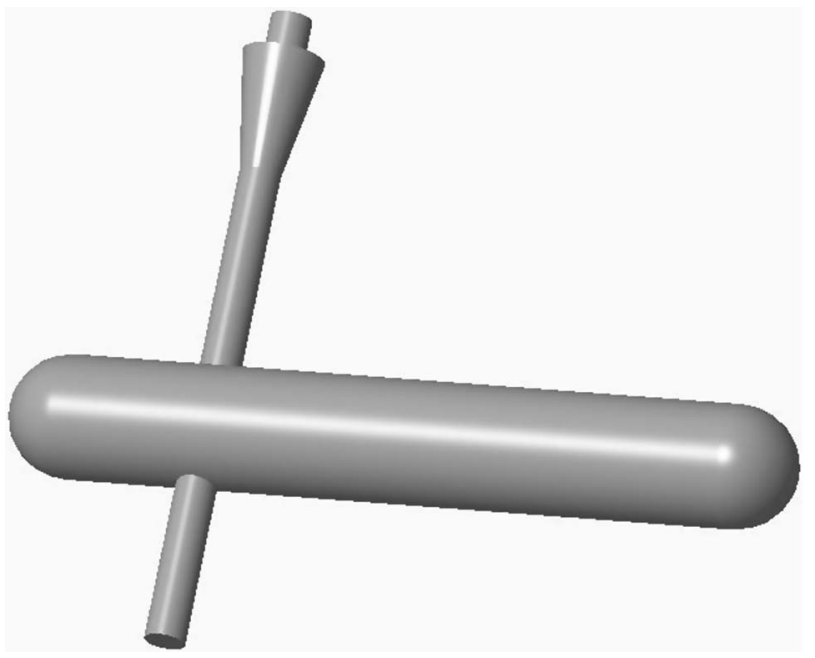

Fig. 12. The manifold with an expansion-chamber muffler.

is installed and the expansion ratio $m=6$, the maximum SPL inside the manifold is $91.1 \mathrm{~dB}$, and the average value is $70.4 \mathrm{~dB}$. When the muffler is installed and the expansion ratio $m=9$, the maximum SPL inside the manifold is $82.2 \mathrm{~dB}$, and the average value is $65.8 \mathrm{~dB}$. When the muffler is installed and the expansion ratio $m=16$, the maximum SPL inside the manifold is $80.6 \mathrm{~dB}$, and the average value is $64.4 \mathrm{~dB}$. As the expansion ratio increases, the silencing effect of the muffler becomes better. For these three types of mufflers, a large expansion ratio means a large expansion cavity, and the large expansion cavity can more effectively cause the reflection of sound waves, reflecting part of the sound wave back to the sound source or reflecting back and forth inside the expansion cavity. Thereby achieving

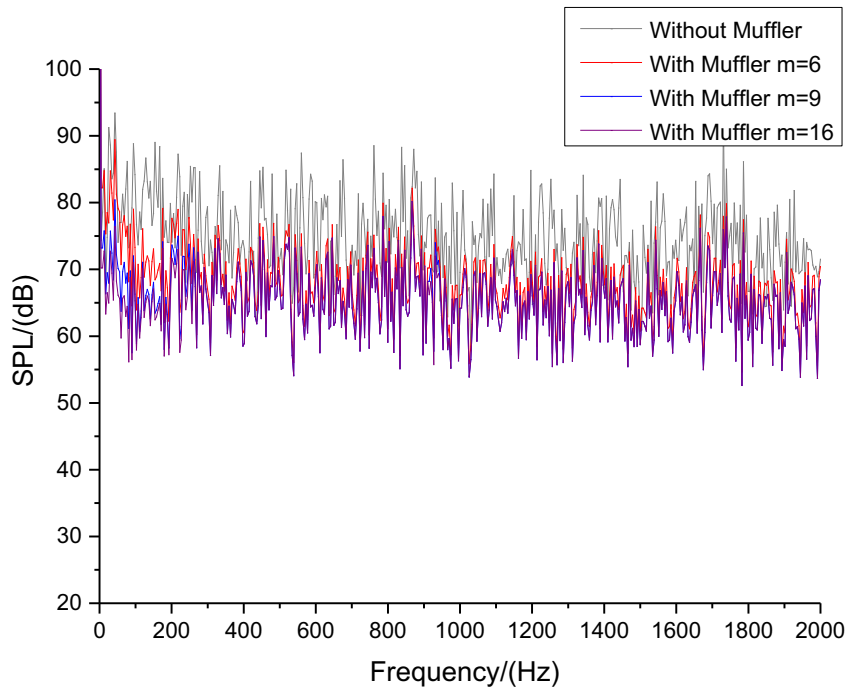

Fig. 13. The SPLs distribution of mufflers.

better purpose of silence. Considering the on-site installation conditions and economy, this paper selects a tapered muffler with an expansion ratio of $m=9$.

To analyze the silencing effect of the muffler with an expansion ratio of $m=9$, the end of the inlet pipe was selected to compare the SPL in the low frequency bands, as shown in Figure 14.

As we can see in Figure 14, the muffler effectively reduces the SPL of the fluid dynamic noise inside the manifold. The reduction of the noise is up to $37.5 \mathrm{~dB}$, and the mean noise reduction is $15.2 \mathrm{~dB}$. It proves that the designed expansion-chamber muffler is performed well in noise reduction. 


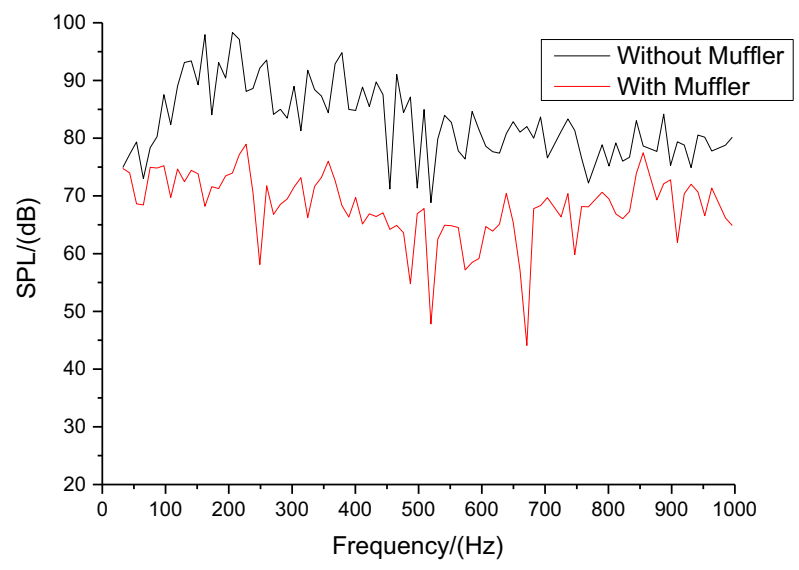

(a) The comparion of low-frequency SPL changes

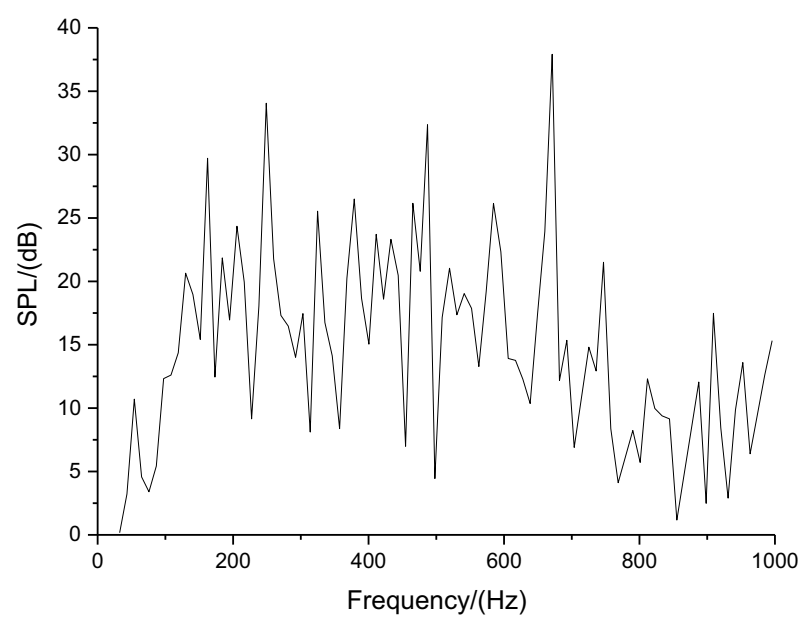

(b) The muffler's effect at low frequency bands

Fig. 14. The silencing effect of the muffler with an expansion ratio of $m=9$. (a) The comparison of low-frequency SPL changes. (b) The muffler's effect at low frequency bands.

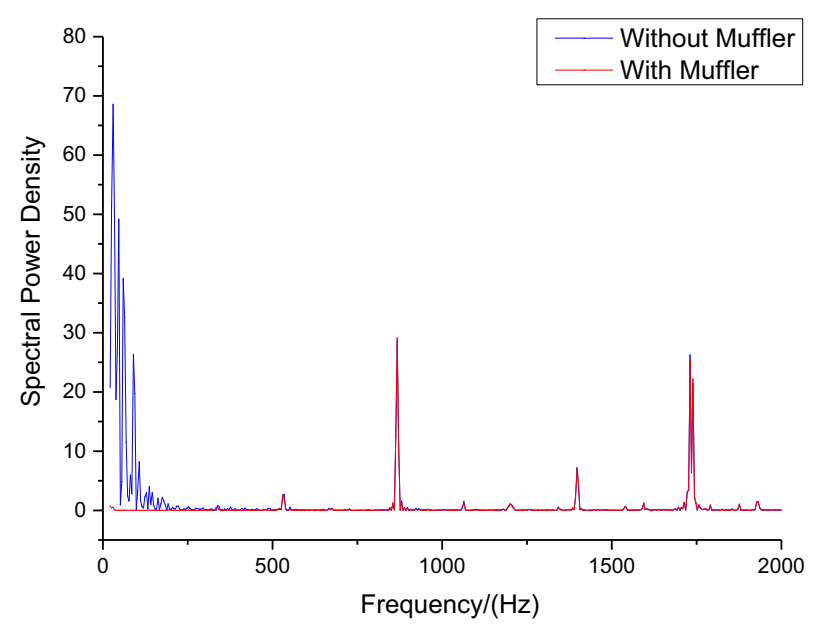

Fig. 15. The spectral power density at the end of the inlet pipe of manifold.
The spectral power densities are shown in Figure 15. It can be seen that the acoustic energy is mainly concentrated between $20 \mathrm{~Hz}$ and $200 \mathrm{~Hz}$ and reaches the peak in a few frequency ranges and that the acoustic energy in other frequency ranges is weak and near zero. At the same time, the acoustic energy in low frequencies is greatly reduced after the muffler is installed, which further proves the excellent noise reduction effect of the expansion chamber muffler.

\section{Conclusion}

The phenomenon of noise pollution exists in many natural gas transmission stations. In this paper, the SPL of manifolds with different number of outlet pipes and different positions of intake pipes are analyzed by ANSYS Fluent, and an expansion-chamber muffler is designed to address the fluid dynamic noise inside the manifold. The results of numerical simulation show that:

(1) The SPL of manifold in natural gas station is relatively high, and even reaches $100 \mathrm{~dB}$ in some frequency bands, which is far beyond the upper limit of $85 \mathrm{~dB}$ of noise emission in the specification.

(2) By changing the number of outlet pipes, the SPL inside the manifold drops significantly. The SPL of the manifold with one inlet and two outlets are reduced by about $10 \mathrm{~dB}$ compared to the manifold with one inlet and one outlet, and the SPL of the manifold with one inlet and three outlets is about $10 \mathrm{~dB}$ lower than that the manifold with one inlet and two outlets.

(3) The different positions of intake pipes affect the SPL inside manifolds. When inlet and outlet piping are staggered, the SPL is $2 \mathrm{~dB} \sim 3 \mathrm{~dB}$ higher than that of the opposite connection for the manifold with one inlet, one outlet and the manifold with one inlet, two outlets, when the inlet pipe is connected correspondingly to the middle outlet pipe, the SPL is lower than the other two structures for the manifold with one inlet, three outlets.

(4) The designed muffler can effectively reduce the SPL of the fluid dynamic noise inside the manifold, especially in low frequencies. The noise reduction is up to $37.5 \mathrm{~dB}$, and the mean noise reduction is $15.2 \mathrm{~dB}$. The maximum noise in the manifold is controlled at about $80 \mathrm{~dB}$, and the average value is controlled at about $60 \mathrm{~dB}$.

Acknowledgments. The authors are grateful for the research support received from Applied Basic Research Program of Sichuan Province (2019YJ0352).

\section{References}

1 Liu E., Li W., Cai H., Peng S. (2019) Formation mechanism of trailing oil in product oil pipeline, Processes 7, 7. doi: $10.3390 /$ pr7010007. 
2 Shi J., Lv L., Wu Y. (2013) Natural gas long distance transmission pipeline station noise control technology, Electr. Design Eng. 21, 79-80, 84. doi: 10.14022/j.cnki. dzsjgc.2013.04.002.

3 Zhu Q., Yan S. (2017) CFD analog-based elbow flow noise study of gas stations, Pipeline Tech. Equip. 2, 19-21. doi: 10.3969/j.issn.1004-9614.2017.02.007.

4 Lighthill M.J. (1952) On sound generated aerodynamically. I. General theory, Proc. R. Soc. London, Ser. A 211, 564-587. doi: 10.1098/rspa.1952.0060.

5 Lighthill M.J. (1954) On sound generated aerodynamically. II. Turbulence as a source of sound, Proc. R. Soc. London, Ser. A 222, 1-32. doi: 10.1098/rspa.1954.0049.

6 Curle N. (1955) The influence of solid boundaries upon aerodynamic sound, Proc. R. Soc. London, Ser. A 231, 505-514. doi: 10.1098/rspa.1955.0191.

7 Powell A. (1964) Theory of vortex sound, J. Acoust. Soc. Am. 36, 177-195. doi: 10.1121/1.1918931.

8 Williams J.E., Hawkings D.L. (1969) Sound generation by turbulence and surfaces in arbitrary motion, Philos. Trans. R. Soc. London, Ser. A 264, 321-342. doi: 10.1098/rsta.1969.0031.

9 Lauchle G.C. (1977) Noise generated by axisymmetric turbulent boundary-layer flow, J. Acoust. Soc. Am. 61, 694-703. doi: 10.1121/1.381356.

10 Goldstein M. (1974) Unified approach to aerodynamic sound generation in the presence of sound boundaries, $J$ Acoust. Soc. Am. 56, 497-509. doi: 10.1121/1.1903283.

11 Ma D., Li P., Dai G., Wang H. (1978) Pressure dependence of turbulent jet noise, Acta Physica Sinica 2, 122-125. doi: 10.7498/aps.27.121.

12 Ma D., Li P., Dai G., Wang H. (1980) Shock associated noise from choked jets, Acta Acust. 3, 172-182. doi: 10.15949/j. cnki.0371-0025.1980.03.002.

13 Ma D. (1987) Development of the law of turbulent jet noise, Acta Acust. 5, 321-328. doi: 10.15949/j.cnki.0371-0025.1987. 05.001.

14 Wu J. (1991) Wave-vortex interactions. I, Adv. Mech. 4, 430-443. doi: 10.6052/1000-0992-1991-4-J1991-050.

$15 \mathrm{Wu}$ J. (1992) Wave-vortex interactions. II, Adv. Mech. 1, 35-46. doi: 10.6052/1000-0992-1992-1-J1992-005.

16 Mankbadi R.R., Hayder M.E., Povinelli L.A. (1994) Structure of supersonic jet flow and its radiated sound, AIAA J. 32, 897-906. doi: 10.2514/3.12072.

17 Lyrintzis A.S., Mankbadi R.R. (1996) Prediction of the farfield jet noise using Kirchhoff's formulation, AIAA J. 1, 1-4. doi: 10.2514/3.13079.
18 Wu S., Zhang W. (2005) Investigated numerically on flowfield of valves and experimental study of valve-noise, Valve $\mathbf{1}$, 7-10. doi: 10.16630/j.cnki.1002-5855.2005.01.004.

19 Tan H., Wang Q. (2015) Numerical simulation of manifold's effect on the velocity field of gas pipeline downstream, Contemp. Chem. Ind. 44, 215-220. doi: 10.13840/j.cnki. cn21-1457/tq.2015.01.070.

20 Liu E., Peng S., Yang T. (2018) Noise-silencing technology for upright venting pipe jet noise, Adv. Mech. Eng. 10, 1-15. doi: $10.1177 / 1687814018794819$.

21 Liu E., Yan S., Peng S., Huang L., Jiang Y. (2016) Noise silencing technology for manifold flow noise based on ANSYS fluent, J. Nat. Gas Sci. Eng. 29, 322-328. doi: 10.1016/j. jngse.2016.01.021.

22 Liu E., Yan S., Peng S., Huang L. (2016) Large Eddy Simulation and FW-H acoustic analogy of flow-induced noise in elbow pipe, J. Comput. Theor. Nanosci. 12, 2866-2873. doi: $10.1166 /$ jctn.2015.4191.

23 Liu E., Lv L., Ma Q., Kuang J., Zhang L. (2019) Steady-state optimization operation of the West-EastGas Pipeline, Adv. Mech. Eng. 11, 1-14. doi: 10.1177/ 1687814018821746.

24 Cao X., Peng W., Luo L., Yan M. (2017) Way to eliminate noise of pressure regulating valve and collection pipe in Gas station, Autom. Instrum. 10, 217-219. doi: 10.14016/j. cnki.1001-9227.2017.10.217.

25 Yan S., Lin Y., Xia Y. (2014) Study on the flow noise in gas pipeline network, Contemp. Chem. Ind. 43, 1806-1808. doi: 10.13840/j.cnki.cn21-1457/tq.2014.09.120.

26 Lu H., Huang K., Fu L., Zhang Z., Wu S., Lyu Y., Zhang X. (2018) Study on leakage and ventilation scheme of gas pipeline in tunnel, J. Nat. Gas Sci. Eng. 53, 347-358. doi: 10.1016/j.jngse.2018.03.019.

27 Li Z., Hou G., Wu C. (2007) Research progress on sound radiation of turbulence in pipeline, Chin. J. Ship Res. 1, 34-38. doi: 10.3969/j.issn.1673-3185.2007.01.007.

28 Lu H., Wu X., Huang K. (2018) Study on the effect of reciprocating pump pipeline system vibration on oil transportation stations, Energies 11, 1, 132. doi: 10.3390/en11010132.

29 Ni M. (2006) Theories and applications of mufflers, Audio Eng. 3, 55-57. doi: 10.16311/j.audioe.2006.03.016.

30 Wang W., Li G., Ni J., Xiao G., Tian Y. (2007) Comparison of interior flow field and pressure field between two different mufflers, Trans. CSICE 1, 34-38. doi: 10.16236/j.cnki. nrjxb.2007.03.014. 